2. G. D. Mostow, Self-adjoint groups, Ann. of Math. vol. 62 (1955) pp. 44-55.

3. H. C. Wang, Discrete subgroups of solvable Lie groups I, Ann. of Math. vol. 64 (1956) pp. 1-19.

4. H. Zassenhaus, Beweis eines Satze über diskrete Gruppen, Abh. Math. Sem. Hansischen Univ. vol. 12 (1938) pp. 289-312.

INDIANA UNIVERSITY

\title{
CONDITIONS IMPLYING CONTINUITY OF FUNCTIONS
}

\section{EDWIN HALFAR}

In the study of functions on certain types of spaces, the question naturally arises as to what additional conditions may imply that the functions are continuous. Several papers, mainly $[2 ; 3 ; 4]$, have considered this problem. In this note, some further results of this type are developed.

To avoid repetition, a function $f$ will be at least on a Hausdorff space $X$ onto a Hausdorff space $Y$ with additional restrictions stated as needed. Also $f$ is compact preserving (connected) if when $K$ is a compact (connected) subset of $X, f(K)$ is a compact (connected) subset of $Y ; f$ has closed point inverses if for each $y \in Y, f^{-1}(y)$ is closed and $f$ is monotone if $f^{-1}(y)$ is connected. The rest of the terminology is standard.

In [1], it was shown that if $X$ is regular, $Y$ compact and if $f$ is closed with closed point inverses, $f$ is continuous. Combining this with Theorem 3.1 of [4], one has the result:

THEOREM 1. If $f$ is a closed monotone connected function on a regular space $X$ onto a compact space $Y$, then $f$ is continuous.

It is easy to see that without the assumption that $Y$ is compact, the conclusion need no longer hold.

THEOREM 2. If $X$ is locally compact, then if $f$ is compact preserving and point inverses are closed, $f$ is continuous.

Consider any point $x \in X$. Since $X$ is locally compact, $x$ has a neighborhood $U_{0}$ with a compact closure $\mathrm{Cl} U_{0}$. Because continuity is a local property, one need only consider $f$ restricted to $\mathrm{Cl} U_{0}$. On $\mathrm{Cl} U_{0}, f$ is closed and $f\left(\mathrm{Cl} U_{0}\right)$ is compact. Hence the conditions of Theorem 3 of [1] are satisfied and $f$ is continuous at $x$.

Received by the editors July 17, 1959. 
If $X$ is not locally compact, then $f$ may not be continuous. The function in the first example on [3, p. 162] is such an instance.

Definition 1. A function $f$ has at worst a removable discontinuity at $x \in X$ if there is a $y \in Y$ such that for each neighborhood $V$ of $y$, there is a neighborhood $U$ of $x$ such that $f(U-[x]) \subset V$.

If $X$ satisfies the first axiom of countability, this definition is equivalent to Definition 3.2 of [4]. With this interpretation, the conditions of Theorem 3.6 of [4] may be relaxed somewhat.

Theorem 3. If $X$ is locally connected and $f$ is connected, then $f$ is continuous at $x_{0}$ if and only if $f$ has at worst a removable discontinuity at $x_{0}$.

With only minor change, the proof given by Pervin and Levine applies here.

TheOREM 4. If $X$ is regular and $f$ is a closed function with closed point inverses, then if $f$ has a removable discontinuity at $x_{0} \in X, f$ is continuous at $x_{0}$.

If $x_{0}$ is isolated in $X$, the result is obviously true. Assume that $x_{0}$ is nonisolated and $f$ is not continuous at $x_{0}$. Let $y$ be the point of $Y$ determined by the hypothesis. Since $y \neq f\left(x_{0}\right)$ and $f^{-1}(y)$ is closed, a neighborhood $U$ of $x_{0}$ exists such that $f^{-1}(y) \cap \mathrm{Cl} U=\varnothing$. Then $y \notin f(\mathrm{Cl} U)$ and because $f(\mathrm{Cl} U)$ is closed, a neighborhood $V$ of $y$ exists for which $V \cap f(\mathrm{Cl} U)=\varnothing$. There is a neighborhood $W$ of $x_{0}$ such that $f\left(W-\left[x_{0}\right]\right) \subset V$. Since $x_{0}$ is nonisolated, $U \cap W-\left[x_{0}\right] \neq \varnothing$. Hence $\varnothing \neq f\left(W-\left[x_{0}\right]\right) \cap f(\mathrm{Cl} U) \subset V \cap f(\mathrm{Cl} U)$, a contradiction.

|Definition 2. A space $X$ will be said to have property $K$ at a point $x$ if for each infinite subset $A$ having $x$ as an accumulation point, there is a compact subset of $A \cup[x]$ which has $x$ as an accumulation point.

Theorem 5. If $X$ has property $K$ at $x_{0}$, then if $f$ is compact preserving and has closed point inverses, $f$ is continuous at $x_{0}$.

It can be assumed that $x_{0}$ is nonisolated. Suppose $f$ is not continuous at $x_{0}$ and that $u$ is the family of neighborhoods of $x_{0}$. Then for some neighborhood $V$ of $f\left(x_{0}\right)$ and for each $U \in \mathcal{u}$, there is an $x_{u}$ such that $x_{u} \in U \cap f^{-1}(Y-V)$. Let $A=\left\{x_{u} \mid U \in \mathcal{u}\right\}$. Then $A$ is infinite, for $x_{0}$ is an accumulation point of $A$. By hypothesis, there is an infinite compact subset $K$ of $A \cup\left[x_{0}\right]$. By Theorem $2, f$ restricted to $K$ is continuous. However $f\left(K-\left[x_{0}\right]\right) \subset Y-V$ but $f\left(x_{0}\right) \in V$, a contradiction. ${ }^{1}$

1 This is the referee's revision of the author's original proof. 
Theorem 6. If $X$ is locally connected with property $K$ at each point and if $f$ is compact preserving and connected, then $f$ is continuous.

It need only be shown that point inverses are closed.

Let $y_{0} \in Y$ and suppose $x_{0} \in \mathrm{Cl} f^{-1}\left(y_{0}\right)-f^{-1}\left(y_{0}\right)$. Denote the family of connected neighborhoods of $x_{0}$ by $\mathfrak{e}$ and the family of neighborhoods of $y_{0}$ by $u$. Select disjoint open neighborhoods $V$ and $U_{0}$ of $f\left(x_{0}\right)$ and $y_{0}$ respectively. For each $C \in \mathcal{C}$ and $U \in \mathcal{U}$, let the point $y(U, C) \in f(C) \cap U \cap U_{0}-\left[y_{0}\right]$ and the point $x(U, C) \in f^{-1}(y(U, C))$ $\cap C$. The set $A$ of all such $x(U, C)$ is infinite and has $x_{0}$ as an accumulation point. By hypothesis, $A \cup\left[x_{0}\right]$ has an infinite compact subset $K$ with $x_{0}$ as an accumulation point. Note that $x_{0} \in K$. Let $g$ denote the function $f$ restricted to $K$. Then $S=g(K)-\left[g\left(x_{0}\right)\right]$ $=g(K) \cap(Y-V)$ is an infinite compact set and must have an accumulation point $z$. If $x=g^{-1}(z)$ is isolated in $K$, then $K-[x]$ and hence $S-[z]$ are compact, a contradiction. Assume then that for each accumulation point of $S$, its inverse in $K$ is an accumulation point of $K$.

Let $A$ be the set of accumulation points of $K$, excluding $x_{0}$. For each $x \in A$, select dis oint open neighborhoods $W_{x}$ and $R_{x}$ of $x$ and $x_{0}$ respectively. Each $K-W_{x}$ is compact and each $B_{x}=g\left(K-W_{x}\right) \cap S$ is a closed non-null subset of $S$. The family $B=\left\{B_{x} \mid x \in A\right\}$ has the finite intersection property, for suppose the contrary. There would exist a finite number of neighborhoods $W_{x_{1}}, \cdots, W_{x_{n}}$ such that $K-\left[x_{0}\right] \subset \bigcup_{i=1}^{i=n} W_{x_{i}}$, but since for each $W_{x_{i}}$, there is a neighborhood $R_{x_{i}}$ of $x_{0}$ disjoint from $W_{x_{i}}, \bigcap_{i=1}^{i=n} R_{x_{i}}$ is a neighborhood of $x_{0}$ disjoint from $\bigcup_{i=1}^{i=n} W_{x_{i}}$, a contradiction. Hence $\cap\left\{B_{x} \mid B_{x} \in \beta\right\} \neq \varnothing$, and for each $y \in \cap\left\{B_{x} \mid B_{x} \in \mathbb{\}}, t=g^{-1}(y)\right.$ is an isolated point of $K$.

Let $T$ be the set of such points $t$ in $K$. Since $T$ is open in $K$, for each $x \in A$ the set $K-\left(W_{x} \cup T\right)$ is compact and non-null. Then $\cap\left\{g\left(K-\left(W_{x} \cup T\right)\right) \mid x \in A\right\} \cap S$ is a null intersection of non-null closed subsets of a compact set, and there is a finite number of neighborhoods $W_{x_{1}}, \cdots, W_{x_{m}}$ which covers $K-\left(T \cup\left[x_{0}\right]\right)$. Since $x_{0}$ is an accumulation point of $K, T$ must be infinite and hence $T \cup\left[x_{0}\right]$ has a compact infinite subset $H$ whose only accumulation point is $x_{0}$. Then $g(H) \cap S$ is an infinite compact subset of $S$ and must have an accumulation point $z$, a contradiction since $g^{-1}(z)$ is an isolated point of $K$.

Thus it follows that $x_{0} \in f^{-1}\left(y_{0}\right)$ and $f^{-1}\left(y_{0}\right)$ is closed. By Theorem $5, f$ is continuous.

Spaces satisfying the first axiom of countability obviously have property $K$. In conclusion there is given an example of a space in 
which property $K$ holds but local countability does not.

Let $X=\cup_{n=-\infty}^{\infty} S_{n}$ be a planar set of points where for

$$
\begin{aligned}
n \geqq 1, \quad S_{n} & =\{(x, y) / 0 \leqq x \leqq n /(n+1), y=x(n+1) / n\} ; \\
n \leqq-1, & S_{n}=\{(x, y) / 0 \geqq x \geqq n /(1-n), y=x(1-n) / n\} \text { and } \\
S_{0} & =\{(x, y) / 0=x, 0 \leqq y \leqq 1\} .
\end{aligned}
$$

For any point $(x, y) \neq(0,0)$, let the neighborhoods of $(x, y)$ be defined by relativization of the Euclidean topology of the plane. At $(0,0)$, define a base as composed of sets of the form $\bigcup_{n=-\infty}^{n=\infty} E_{n}$, where for each integer $n, E_{n} \subset S_{n}$ is a half-open interval with $(0,0)$ as the endpoint. Then the first axiom of countability is not satisfied at $(0,0)$, but the space does have property $K$ there.

\section{REFERENCES}

1. E. Halfar, Compact mappings, Proc. Amer. Math. Soc. vol. 8 (1957) pp. 828830.

2. V. L. Klee and W. R. Utz, Some remarks on continuous transformations, Proc. Amer. Math. Soc. vol. 5 (1954) pp. 182-184.

3. Ky Fan and R. A. Struble, Continuity in terms of connectedness, Nederl. Akad. Wetensch. Proc. Ser. A vol. 57 (1954) pp. 161-164.

4. W. J. Pervin and N. Levine, Connected mappings of Hausdorff spaces, Proc. Amer. Math. Soc. vol. 9 (1958) pp. 488-495.

UNIVERSITY OF NEBRASKA 